\title{
ORIGINAL PAPER \\ Existence, Comparison, and Convergence Results for a Class of Elliptic Hemivariational Inequalities
}

\author{
Claudia M. Gariboldi ${ }^{1}$. Stanisław Migórski ${ }^{2,3} \cdot$ Anna Ochal $^{3}$ (1) \\ Domingo A. Tarzia ${ }^{4}$
}

Accepted: 23 June 2021 / Published online: 9 July 2021

(c) The Author(s) 2021

\begin{abstract}
In this paper we study a class of elliptic boundary hemivariational inequalities which originates in the steady-state heat conduction problem with nonmonotone multivalued subdifferential boundary condition on a portion of the boundary described by the Clarke generalized gradient of a locally Lipschitz function. First, we prove a new existence result for the inequality employing the theory of pseudomonotone operators. Next, we give a result on comparison of solutions, and provide sufficient conditions that guarantee the asymptotic behavior of solution, when the heat transfer coefficient tends to infinity. Further, we show a result on the continuous dependence of solution on the internal energy and heat flux. Finally, some examples of convex and nonconvex potentials illustrate our hypotheses.
\end{abstract}

Keywords Elliptic hemivariational inequality · Asymptotic behavior · Clarke generalized gradient $\cdot$ Mixed problem $\cdot$ Convergence $\cdot$ Nonlinear elliptic equation

Mathematics Subject Classification 35J05 · 35J65 · 35J87 · 49J45

\section{Introduction}

We consider a bounded domain $\Omega$ in $\mathbb{R}^{d}$ whose regular boundary $\Gamma$ consists of the union of three disjoint portions $\Gamma_{i}, i=1,2,3$ with $\left|\Gamma_{i}\right|>0$, where $\left|\Gamma_{i}\right|$ denotes the

\footnotetext{
This project has received funding from the European Union's Horizon 2020 Research and Innovation Programme under the Marie Skłodowska-Curie Grant Agreement No. 823731 CONMECH. It is supported by NSF of Guangxi, Grant No.: 2018GXNSFAA281353, Beibu Gulf University Project No. 2018KYQD06, and the projects financed by the Ministry of Science and Higher Education of Republic of Poland under Grants Nos. 4004/GGPJII/H2020/2018/0 and 440328/PnH2/2019. The first and the fourth authors are also partially sponsored by the Project PIP No. 0275 from CONICET-UA, Rosario, Argentina.
}

Anna Ochal

anna.ochal@uj.edu.pl

Extended author information available on the last page of the article 
$(d-1)$-dimensional Hausdorff measure of the portion $\Gamma_{i}$ on $\Gamma$. The outward normal vector on the boundary is denoted by $n$. We formulate the following two steady-state heat conduction problems with mixed boundary conditions:

$$
\begin{array}{llll}
-\Delta u=g \text { in } \Omega, & \left.u\right|_{\Gamma_{1}}=0, & -\left.\frac{\partial u}{\partial n}\right|_{\Gamma_{2}}=q, & \left.u\right|_{\Gamma_{3}}=b, \\
-\Delta u=g \text { in } \Omega, & \left.u\right|_{\Gamma_{1}}=0, & -\left.\frac{\partial u}{\partial n}\right|_{\Gamma_{2}}=q, & -\left.\frac{\partial u}{\partial n}\right|_{\Gamma_{3}}=\alpha(u-b),
\end{array}
$$

where $u$ is the temperature in $\Omega, g$ is the internal energy in $\Omega, b$ is the temperature on $\Gamma_{3}$ for (1) and the temperature of the external neighborhood of $\Gamma_{3}$ for (2), $q$ is the heat flux on $\Gamma_{2}$ and $\alpha>0$ is the heat transfer coefficient on $\Gamma_{3}$, which satisfy the hypothesis: $g \in L^{2}(\Omega), q \in L^{2}\left(\Gamma_{2}\right)$ and $b \in H^{\frac{1}{2}}\left(\Gamma_{3}\right)$.

Throughout the paper we use the following notation

$$
\begin{aligned}
& V=H^{1}(\Omega), \quad V_{0}=\left\{v \in V \mid v=0 \text { on } \Gamma_{1}\right\}, \\
& K=\left\{v \in V \mid v=0 \text { on } \Gamma_{1}, v=b \text { on } \Gamma_{3}\right\}, \\
& K_{0}=\left\{v \in V \mid v=0 \text { on } \Gamma_{1} \cup \Gamma_{3}\right\}, \\
& a(u, v)=\int_{\Omega} \nabla u \nabla v d x, \quad a_{\alpha}(u, v)=a(u, v)+\alpha \int_{\Gamma_{3}} \gamma u \gamma v d \Gamma, \\
& L(v)=\int_{\Omega} g v d x-\int_{\Gamma_{2}} q \gamma v d \Gamma, \quad L_{\alpha}(v)=L(v)+\alpha \int_{\Gamma_{3}} b \gamma v d \Gamma,
\end{aligned}
$$

where $\gamma: V \rightarrow L^{2}(\Gamma)$ denotes the trace operator on $\Gamma$. In what follows, we write $u$ for the trace of a function $u \in V$ on the boundary. In a standard way, we obtain the following variational formulations of (1) and (2), respectively:

find $u_{\infty} \in K$ such that $a\left(u_{\infty}, v\right)=L(v)$ for all $v \in K_{0}$,

find $u_{\alpha} \in V_{0}$ such that $a_{\alpha}\left(u_{\alpha}, v\right)=L_{\alpha}(v)$ for all $v \in V_{0}$.

The standard norms on $V$ and $V_{0}$ are denoted by

$$
\begin{gathered}
\|v\|_{V}=\left(\|v\|_{L^{2}(\Omega)}^{2}+\|\nabla v\|_{L^{2}\left(\Omega ; \mathbb{R}^{d}\right)}^{2}\right)^{1 / 2} \text { for } v \in V, \\
\|v\|_{V_{0}}=\|\nabla v\|_{L^{2}\left(\Omega ; \mathbb{R}^{d}\right)} \text { for } v \in V_{0} .
\end{gathered}
$$

It is well known by the Poincare inequality, see [5, Proposition 2.94], that on $V_{0}$ the above two norms are equivalent. Note that the form $a$ is bilinear, symmetric, continuous and coercive with constant $m_{a}>0$, i.e.

$$
a(v, v)=\|v\|_{V_{0}}^{2} \geq m_{a}\|v\|_{V}^{2} \text { for all } v \in V_{0}
$$

It is well known that the regularity of solution to the mixed elliptic problems (1) and (2) is problematic in the neighborhood of a part of the boundary, see for example 
the monograph [12]. Regularity results for elliptic problems with mixed boundary conditions can be found in $[1,2,14]$. Moreover, sufficient hypothesis on the data in order to have $H^{2}$ regularity for elliptic variational inequalities is given in [23]. We remark that, under additional hypotheses on the data $g, q$ and $b$, problems (1) and (2) can be considered as steady-state two phase Stefan problems, see, for example, $[10,26,28,30]$.

Problems (3) and (4) have been extensively studied in several papers such as [10,2629]. Some properties of monotonicity and convergence, when the parameter $\alpha$ goes to infinity, obtained in the aforementioned works, are recalled in the following result.

Theorem 1 If the data satisfy $b=$ const. $>0, g \in L^{2}(\Omega)$ and $q \in L^{2}\left(\Gamma_{2}\right)$ with the properties $q \geq 0$ on $\Gamma_{2}$ and $g \leq 0$ in $\Omega$, then

(i) $u_{\infty} \leq b$ in $\Omega$,

(ii) $u_{\alpha} \leq b$ in $\Omega$,

(iii) $u_{\alpha} \leq u_{\infty}$ in $\Omega$,

(iv) if $\alpha_{1} \leq \alpha_{2}$, then $u_{\alpha_{1}} \leq u_{\alpha_{2}}$ in $\Omega$,

(v) $u_{\alpha} \rightarrow u_{\infty}$ in $V$, as $\alpha \rightarrow \infty$.

The main goal of this paper is to study a generalization of problem (2) for which we provide sufficient conditions that guarantee the comparison properties and asymptotic behavior, as $\alpha \rightarrow \infty$, stated in Theorem 1. Moreover, for a more general problem, we also show a result on the continuous dependence of solution on the data $g$ and $q$.

The mixed nonlinear boundary value problem for the elliptic equation under consideration reads as follows.

$$
-\Delta u=g \text { in } \Omega,\left.\quad u\right|_{\Gamma_{1}}=0, \quad-\left.\frac{\partial u}{\partial n}\right|_{\Gamma_{2}}=q, \quad-\left.\frac{\partial u}{\partial n}\right|_{\Gamma_{3}} \in \alpha \partial j(u) .
$$

Here $\alpha$ is a positive constant while the function $j: \Gamma_{3} \times \mathbb{R} \rightarrow \mathbb{R}$, called a superpotential (nonconvex potential), is such that $j(x, \cdot)$ is locally Lipschitz for a.e. $x \in \Gamma_{3}$ and not necessarily differentiable. Since in general $j(x, \cdot)$ is nonconvex, so the multivalued condition on $\Gamma_{3}$ in problem (6) is described by a nonmonotone relation expressed by the generalized gradient of Clarke. Such multivalued relation in problem (6) is met in certain types of steady-state heat conduction problems (the behavior of a semipermeable membrane of finite thickness, a temperature control problems, etc.). Further, problem (6) can be considered as a prototype of several boundary semipermeability models, see [15,19,20,32], which are motivated by problems arising in hydraulics, fluid flow problems through porous media, and electrostatics, where a solution represents the pressure and the electric potentials. Note that the analogous problems with maximal monotone multivalued boundary relations (that is the case when $j(x, \cdot)$ is a convex function) were considered in [3,9], see also references therein.

Under the above notation, the weak formulation of the elliptic problem (6) becomes the following boundary hemivariational inequality:

$$
\text { find } u \in V_{0} \text { such that } a(u, v)+\alpha \int_{\Gamma_{3}} j^{0}(u ; v) d \Gamma \geq L(v) \text { for all } v \in V_{0} \text {. }
$$


Here and in what follows we often omit the variable $x$ and we simply write $j(r)$ instead of $j(x, r)$. Observe that if $j(x, \cdot)$ is a convex function for a.e. $x \in \Gamma_{3}$, then problem (7) reduces to the variational inequality of second kind:

$$
\begin{aligned}
& \text { find } u \in V_{0} \text { such that } a(u, v-u)+\alpha \int_{\Gamma_{3}}(j(v)-j(u)) d \Gamma \\
& \quad \geq L(v-u) \text { for all } v \in V_{0} .
\end{aligned}
$$

Note that when $j(r)=\frac{1}{2}(r-b)^{2}$, problem (8) reduces to a variational inequality corresponding to problem (2). Several other examples of convex potentials can be found in various diffusion problems. For instance, the following convex functions:

$$
j(r)=|r|, \quad j(r)=\left\{\begin{array}{ll}
\beta(r-c)^{5} & \text { if } r \geq c, \\
0 & \text { if } r<c,
\end{array} \text { and } j(r)= \begin{cases}\beta r^{9 / 4} & \text { if } r \geq 0, \\
0 & \text { if } r<0\end{cases}\right.
$$

with suitable constants $\beta>0$ and $c \in \mathbb{R}$, appear in models which describe a free boundary problem with the Tresca condition, see [4], the Stefan-Boltzman heat radiation law, and the natural convection, respectively, see [3,13], and the references therein for further applications and extensions. On the other hand, the stationary heat conduction models with nonmonotone multivalued subdifferential interior and boundary semipermeability relations can not be described by convex potentials. They use locally Lipschitz potentials and their weak formulations lead to hemivariational inequalities, see [19, Chapter 5.5.3] and [20].

We mention that the theory of hemivariational and variational inequalities has been proposed in the 1980s by Panagiotopoulos, see [19,21,22], as variational formulations of important classes of inequality problems in mechanics. In the last few years, new kinds of variational, hemivariational, and variational-hemivariational inequalities have been investigated, see recent monographs $[5,17,25]$, and the theory has emerged today as a new and interesting branch of applied mathematics.

The rest of the paper is structured as follows. In Sect. 2 we provide a new existence result for problem (7). In Sect. 3 we establish two comparison properties for solutions to problem (7). The result on convergence of solutions of problem (7) to the solution of problem (3), when the parameter $\alpha$ goes to infinity, is provided in Sect. 4. In Sect. 5 we study the continuous dependence of a solution to problem (7) on the internal energy $g$ and the heat flux $q$. The proofs are based on arguments of compactness, lower semicontinuity, monotonicity, various estimates, the theory of elliptic hemivariational inequalities and nonsmooth analysis [6-9,11,17,22,24,25,31]. Finally, in Sect. 6 we deliver several examples of convex and nonconvex potentials which satisfy the hypotheses on the function $j$ required in this paper.

\section{Preliminaries}

In this section first recall standard notation and preliminary concepts, and then provide a new result on existence of solution to the elliptic hemivariational inequality (7). 
Let $\left(X,\|\cdot\|_{X}\right)$ be a Banach space, $X^{*}$ be its dual, and $\langle\cdot, \cdot\rangle$ denote the duality between $X^{*}$ and $X$. For a real valued function defined on $X$, we have the following definitions, see [6, Sect. 2.1] and [7,17].

Definition 2 A function $\varphi: X \rightarrow \mathbb{R}$ is said to be locally Lipschitz, if for every $x \in X$ there exist $U_{x}$ a neighborhood of $x$ and a constant $L_{x}>0$ such that

$$
|\varphi(y)-\varphi(z)| \leq L_{x}\|y-z\|_{X} \text { for all } y, z \in U_{x} .
$$

For such a function the generalized (Clarke) directional derivative of $j$ at the point $x \in X$ in the direction $v \in X$ is defined by

$$
\varphi^{0}(x ; v)=\limsup _{y \rightarrow x, \lambda \rightarrow 0^{+}} \frac{\varphi(y+\lambda v)-\varphi(y)}{\lambda} .
$$

The generalized gradient (subdifferential) of $\varphi$ at $x$ is a subset of the dual space $X^{*}$ given by

$$
\partial \varphi(x)=\left\{\zeta \in X^{*} \mid \varphi^{0}(x ; v) \geq\langle\zeta, v\rangle \text { for all } v \in X\right\}
$$

We shall use the following properties of the generalized directional derivative and the generalized gradient, see [17, Proposition 3.23].

Proposition 3 Assume that $\varphi: X \rightarrow \mathbb{R}$ is a locally Lipschitz function. Then the following hold:

(i) for every $x \in X$, the function $X \ni v \mapsto \varphi^{0}(x ; v) \in \mathbb{R}$ is positively homogeneous, and subadditive, i.e.,

$$
\begin{aligned}
& \varphi^{0}(x ; \lambda v)=\lambda \varphi^{0}(x ; v) \text { for all } \lambda \geq 0, v \in X, \\
& \varphi^{0}\left(x ; v_{1}+v_{2}\right) \leq \varphi^{0}\left(x ; v_{1}\right)+\varphi^{0}\left(x ; v_{2}\right) \text { for all } v_{1}, v_{2} \in X,
\end{aligned}
$$

respectively.

(ii) for every $x \in X$, we have $\varphi^{0}(x ; v)=\max \{\langle\zeta, v\rangle \mid \zeta \in \partial \varphi(x)\}$.

(iii) the function $X \times X \ni(x, v) \mapsto \varphi^{0}(x ; v) \in \mathbb{R}$ is upper semicontinuous.

(iv) for every $x \in X$, the gradient $\partial \varphi(x)$ is a nonempty, convex, and weakly * compact subset of $X^{*}$.

(v) the graph of the generalized gradient $\partial \varphi$ is closed in $X \times\left(\right.$ weak $\left.{ }^{*}-X^{*}\right)$-topology.

Now, we pass to a result on existence of solution to the elliptic hemivariational inequality:

find $u \in V_{0}$ such that $a(u, v)+\alpha \int_{\Gamma_{3}} j^{0}(u ; v) d \Gamma \geq f(v)$ for all $v \in V_{0}$.

We admit the following standing hypothesis.

$H(j): j: \Gamma_{3} \times \mathbb{R} \rightarrow \mathbb{R}$ is such that 
(a) $j(\cdot, r)$ is measurable for all $r \in \mathbb{R}$,

(b) $j(x, \cdot)$ is locally Lipschitz for a.e. $x \in \Gamma_{3}$,

(c) there exist $c_{0}, c_{1} \geq 0$ such that $|\partial j(x, r)| \leq c_{0}+c_{1}|r|$ for all $r \in \mathbb{R}$, a.e. $x \in \Gamma_{3}$,

(d) $j^{0}(x, r ; b-r) \leq 0$ for all $r \in \mathbb{R}$, a.e. $x \in \Gamma_{3}$ with a constant $b \in \mathbb{R}$.

Here the constant $b$ in $H(j)(\mathrm{d})$ is the same as in the boundary condition on $\Gamma_{3}$ (see (1) or (2)).

Note that the existence results for elliptic hemivariational inequalities can be found in several contributions, see [5,16-19]. In comparison to other works, the new hypothesis is $H(j)(\mathrm{d})$. Under this condition we will show both existence of a solution to problem (9) and a convergence result when $\alpha \rightarrow \infty$. We underline that, if the hypothesis $H(j)(\mathrm{d})$ is replaced by the relaxed monotonicity condition (see Remark 10 for details)

$$
j^{0}(x, r ; s-r)+j^{0}(x, s ; r-s) \leq m_{j}|r-s|^{2}
$$

for all $r, s \in \mathbb{R}$, a.e. $x \in \Gamma_{3}$ with $m_{j} \geq 0$, and the following smallness condition

$$
m_{a}>\alpha m_{j}\|\gamma\|^{2}
$$

is assumed, then problem (9) is uniquely solvable, see [18, Lemma 20] for the proof. However, this smallness condition is not suitable in the study of problem (9) since for a sufficiently large value of $\alpha$, it is not satisfied.

In the following result we apply a surjectivity result in [17, Proposition 3.61] and partially follow arguments of [18, Lemma 20]. For completeness we provide the proof.

Theorem 4 If $H(j)$ holds, $f \in V_{0}^{*}$ and $\alpha>0$, then the hemivariational inequality (9) has a solution.

Proof Let $\langle\cdot, \cdot\rangle$ stand for the duality pairing between $V_{0}^{*}$ and $V_{0}$. Let $A: V_{0} \rightarrow V_{0}^{*}$ be defined by

$$
\langle A u, v\rangle=a(u, v) \text { for } u, v \in V_{0} .
$$

It is obvious that the operator $A$ is linear, bounded and coercive, i.e., $\langle A v, v\rangle \geq\|v\|_{V_{0}}^{2}$ for all $v \in V_{0}$. Moreover, let $J: L^{2}\left(\Gamma_{3}\right) \rightarrow \mathbb{R}$ be given by

$$
J(w)=\int_{\Gamma_{3}} j(x, w(x)) d \Gamma \text { for all } w \in L^{2}\left(\Gamma_{3}\right) .
$$

From $H(j)(\mathrm{a})-(\mathrm{c})$, by [17, Corollary 4.15], we infer that the functional $J$ enjoys the following properties:

(p1) $J$ is well defined and Lipschitz continuous on bounded subsets of $L^{2}\left(\Gamma_{3}\right)$, hence also locally Lipschitz,

(p2) $J^{0}(w ; z) \leq \int_{\Gamma_{3}} j^{0}(x, w(x) ; z(x)) d \Gamma$ for all $w, z \in L^{2}\left(\Gamma_{3}\right)$, 
(p3) $\|\partial J(w)\|_{L^{2}\left(\Gamma_{3}\right)} \leq \bar{c}_{0}+\bar{c}_{1}\|w\|_{L^{2}\left(\Gamma_{3}\right)}$ for all $w \in L^{2}\left(\Gamma_{3}\right)$ with $\bar{c}_{0}, \bar{c}_{1} \geq 0$.

We introduce the operator $B: V_{0} \rightarrow 2^{V_{0}^{*}}$ defined by

$$
B v=\alpha \gamma^{*} \partial J(\gamma v) \text { for all } v \in V_{0}
$$

where $\gamma^{*}: L^{2}(\Gamma) \rightarrow V_{0}^{*}$ denotes the adjoint to the trace $\gamma$.

We show that $B$ is pseudomonotone and bounded from $V_{0}$ to $2^{V_{0}^{*}}$, see [17, Definition 3.57]. By Proposition 3 (iv), it follows that the values of $\partial J$ are nonempty, convex and weakly compact subsets of $L^{2}\left(\Gamma_{3}\right)$. Hence, the set $B v$ is nonempty, closed and convex in $V_{0}^{*}$ for all $v \in V_{0}$. The operator $B$ is bounded which is a consequence of the following estimate

$$
\|B v\|_{V_{0}^{*}} \leq \alpha\left\|\gamma^{*}\right\|\|\partial J(\gamma v)\|_{L^{2}\left(\Gamma_{3}\right)} \leq \alpha\left\|\gamma^{*}\right\|\left(\bar{c}_{0}+\bar{c}_{1}\|\gamma\|\|v\|_{V_{0}}\right) \text { for all } v \in V_{0} \text {, }
$$

where $\|\gamma\|$ denotes the norm of the trace operator. In order to establish pseudomonotonicity of the operator $B$, we take into account [17, Proposition 3.58(ii)], and prove that $B$ is generalized pseudomonotone.

Let $v_{n}, v \in V_{0}, v_{n} \rightarrow v$ weakly in $V_{0}, v_{n}^{*}, v^{*} \in V_{0}^{*}, v_{n}^{*} \rightarrow v^{*}$ weakly in $V_{0}^{*}$, $v_{n}^{*} \in B v_{n}$ and $\lim \sup \left\langle v_{n}^{*}, v_{n}-v\right\rangle \leq 0$. We show that

$$
v^{*} \in B v \text { and }\left\langle v_{n}^{*}, v_{n}\right\rangle \rightarrow\left\langle v^{*}, v\right\rangle .
$$

From condition $v_{n}^{*} \in B v_{n}$, it follows $v_{n}^{*}=\alpha \gamma^{*} \eta_{n}$ with $\eta_{n} \in \partial J\left(\gamma v_{n}\right)$. By the estimate (p3), it is clear that $\left\{\eta_{n}\right\}$ remains in a bounded subset of $L^{2}\left(\Gamma_{3}\right)$. Thus, at least for a subsequence, denoted in the same way, we may suppose that $\eta_{n} \rightarrow \eta$ weakly in $L^{2}\left(\Gamma_{3}\right)$ with $\eta \in L^{2}\left(\Gamma_{3}\right)$. Using the compactness of the trace operator, we have $\gamma v_{n} \rightarrow \gamma v$ in $L^{2}\left(\Gamma_{3}\right)$ Now, we employ the strong-weak closedness of the graph of $\partial J$, see Proposition $3(\mathrm{v})$, to obtain $\eta \in \partial J(\gamma v)$. On the other hand, by $v_{n}^{*}=\alpha \gamma^{*} \eta_{n}$, it follows $v^{*}=\alpha \gamma^{*} \eta$. Hence, we get $v^{*} \in \alpha \gamma^{*} \partial J(\gamma v)=B v$. Now, it is obvious that

$$
\left\langle v_{n}^{*}, v_{n}\right\rangle=\alpha\left\langle\eta_{n}, \gamma v_{n}\right\rangle_{L^{2}\left(\Gamma_{3}\right)} \longrightarrow \alpha\langle\eta, \gamma v\rangle_{L^{2}\left(\Gamma_{3}\right)}=\left\langle\alpha \gamma^{*} \eta, v\right\rangle=\left\langle v^{*}, v\right\rangle
$$

This completes the proof that $B$ is generalized pseudomonotone. Hence, the operator $B$ is also pseudomonotone.

Subsequently, we note that $A: V_{0} \rightarrow V_{0}^{*}$ is pseudomonotone, see [17, Theorem 3.69], since it is linear, bounded and nonnegative. Therefore, $A$ is pseudomonotone and bounded as a multivalued operator from $V_{0}$ to $2^{V_{0}^{*}}$, see $[17$, Sect. 3.4]. Since the sum of multivalued pseudomonotone operators remains pseudomonotone, see [17, Proposition 3.59 (ii)], we infer that $A+B$ is bounded and pseudomonotone.

Next, we prove that the operator $A+B$ is coercive. In view of the coercivity of $A$, it is enough to show that

$$
\langle B v, v\rangle \geq-d_{0}-d_{1}\|v\|_{V_{0}} \text { for all } v \in V_{0}
$$


with $d_{0}, d_{1} \geq 0$. First, from hypothesis $H(j)(\mathrm{d})$, by Proposition 3 (i)-(ii), we have

$$
\begin{aligned}
j^{0}(x, r ;-r) & =j^{0}(x, r ; b-r-b) \leq j^{0}(x, r ; b-r)+j^{0}(x, r ;-b) \\
& \leq j^{0}(x, r ;-b) \leq|\partial j(x, r)||-b| \leq|b|\left(c_{0}+c_{1}|r|\right)
\end{aligned}
$$

for all $r \in \mathbb{R}$, a.e. $x \in \Gamma_{3}$. Next, let $v \in V_{0}, v^{*} \in B v$. Thus, $v^{*}=\alpha \gamma^{*} \eta$ with $\eta \in \partial J(\gamma v)$. Hence, by the definition of the generalized gradient and the property (p2), we obtain

$$
\begin{aligned}
\alpha\langle\eta,-\gamma v\rangle_{L^{2}\left(\Gamma_{3}\right)} & \leq \alpha J^{0}(\gamma v ;-\gamma v) \leq \alpha \int_{\Gamma_{3}} j^{0}(\gamma v ;-\gamma v) d \Gamma \\
& \leq \alpha|b| \int_{\Gamma_{3}}\left(c_{0}+c_{1}|\gamma v(x)|\right) d \Gamma \leq d_{0}+d_{1}\|v\|_{V_{0}}
\end{aligned}
$$

with $d_{0}, d_{1} \geq 0$. Using the latter and the equality

$$
\alpha\langle\eta, \gamma v\rangle_{L^{2}\left(\Gamma_{3}\right)}=\left\langle\alpha \gamma^{*} \eta, v\right\rangle=\left\langle v^{*}, v\right\rangle
$$

we deduce

$$
\left\langle v^{*}, v\right\rangle \geq-d_{0}-d_{1}\|v\|_{V_{0}} \text { for all } v \in V_{0}
$$

which proves (10). In consequence, we have

$$
\langle(A+B) v, v\rangle \geq\|v\|_{V_{0}}^{2}-d_{1}\|v\|_{V_{0}}-d_{0} .
$$

We conclude that the multivalued operator $A+B$ is bounded, pseudomonotone, and coercive, hence surjective, see [17, Proposition 3.61]. We infer that there exists $u \in V_{0}$ such that $(A+B) u \ni f$.

In the final step of the proof, we observe that any solution $u \in V_{0}$ to the inclusion $(A+B) u \ni f$ is a solution to problem (9). Indeed, we have

$$
A u+\alpha \gamma^{*} \eta=f \text { with } \eta \in \partial J(\gamma u)
$$

and hence

$$
\langle A u, v\rangle+\alpha\langle\eta, \gamma v\rangle_{L^{2}\left(\Gamma_{3}\right)}=\langle f, v\rangle
$$

for all $v \in V_{0}$. Combining the latter with the definition of the generalized gradient and the property (p2), we obtain

$$
\begin{aligned}
\langle f, v\rangle & =\langle A u, v\rangle+\alpha\langle\eta, \gamma v\rangle_{L^{2}\left(\Gamma_{3}\right)} \leq\langle A u, v\rangle+\alpha J^{0}(\gamma u ; \gamma v) \\
& \leq a(u, v)+\alpha \int_{\Gamma_{3}} j^{0}(\gamma u ; \gamma v) d \Gamma
\end{aligned}
$$


for all $v \in V_{0}$. This means that $u \in V_{0}$ solves problem (9). This completes the proof.

\section{Comparison Results}

In this section we study the following two problems under the standing hypothesis $H(j)$ on the superpotential.

For every $\alpha>0$, we consider the hemivariational inequality of the form

$$
\text { find } u \in V_{0} \text { such that } a(u, v)+\alpha \int_{\Gamma_{3}} j^{0}(u ; v) d \Gamma \geq L(v) \text { for all } v \in V_{0}
$$

and the weak form of the elliptic equation

$$
\text { find } u_{\infty} \in K \text { such that } a\left(u_{\infty}, v\right)=L(v) \text { for all } v \in K_{0} \text {. }
$$

Recall that

$$
K=\left\{v \in V \mid v=0 \text { on } \Gamma_{1}, v=b \text { on } \Gamma_{3}\right\}, \quad K_{0}=\left\{v \in V \mid v=0 \text { on } \Gamma_{1} \cup \Gamma_{3}\right\} .
$$

It follows from Theorem 4 that for each $\alpha>0$, problem (11) has a solution $u_{\alpha} \in V_{0}$ while [5, Corollary 2.102] entails that problem (12) has a unique solution $u_{\infty} \in K$. Moreover, it is easy to observe that problem (12) can be equivalently formulated as follows

$$
\text { find } u_{\infty} \in K \text { such that } a\left(u_{\infty}, v-u_{\infty}\right)=L\left(v-u_{\infty}\right) \text { for all } v \in K \text {. }
$$

In what follows we need the hypothesis on the data.

$\left(H_{0}\right): \quad g \in L^{2}(\Omega), g \leq 0$ in $\Omega, q \in L^{2}\left(\Gamma_{2}\right), q \geq 0$ on $\Gamma_{2}$.

Theorem 5 If $H(j),\left(H_{0}\right)$ hold and $b \geq 0$, then

(a) $u_{\alpha} \leq b$ in $\Omega$,

(b) $u_{\alpha} \leq u_{\infty}$ in $\Omega$,

where $u_{\alpha} \in V_{0}$ is a solution to problem (11) and $u_{\infty} \in K$ is the unique solution to problem (12).

Proof (a) Let $w=u_{\alpha}-b$. We shall prove that $w^{+}=0$, where $r^{+}=\max \{0, r\}$ for $r \in \mathbb{R}$. Since $\left.w\right|_{\Gamma_{1}}=-b \leq 0$, we have $\left.w^{+}\right|_{\Gamma_{1}}=0$. We choose $v=-w^{+} \in V_{0}$ in problem (11) to get

$$
a\left(u_{\alpha},-w^{+}\right)+\alpha \int_{\Gamma_{3}} j^{0}\left(u_{\alpha} ;-w^{+}\right) d \Gamma \geq L\left(-w^{+}\right) .
$$

By the linearity of the form $a$, we easily obtain

$$
a\left(u_{\alpha},-w^{+}\right)=-a\left(w^{+}, w^{+}\right),
$$


while $\left(H_{0}\right)$ implies $L\left(w^{+}\right) \leq 0$. Hence

$$
-a\left(w^{+}, w^{+}\right)+\alpha \int_{\Gamma_{3}} j^{0}\left(u_{\alpha} ;-w^{+}\right) d \Gamma \geq L\left(-w^{+}\right) \geq 0,
$$

and

$$
a\left(w^{+}, w^{+}\right) \leq \alpha \int_{\Gamma_{3}} j^{0}\left(u_{\alpha} ;-\left(u_{\alpha}-b\right)^{+}\right) d \Gamma .
$$

Subsequently, $H(j)(\mathrm{d})$ entails

$$
j^{0}\left(x, r ;-(r-b)^{+}\right) \leq 0 \text { for all } r \in \mathbb{R} \text {, a.e. } x \in \Gamma_{3} .
$$

Indeed, if $r \leq b$, then $(r-b)^{+}=0$ and $j^{0}\left(x, r ;-(r-b)^{+}\right)=j^{0}(x, r ; 0)=$ $0 \leq 0$. If $r>b$, we would have $(r-b)^{+}=r-b$ and $j^{0}\left(x, r ;-(r-b)^{+}\right)=$ $j^{0}(x, r ; b-r) \leq 0$. Using the coercivity condition (5) of the form $a$ and (14), we deduce $m_{a}\left\|w^{+}\right\|_{V}^{2} \leq 0$. Hence $w^{+}=0$ in $\Omega$, and finally $u_{\alpha} \leq b$ in $\Omega$.

(b) We denote $w=u_{\alpha}-u_{\infty}$. It is enough to show that $w^{+}=0$ in $\Omega$. We observe that $\left.w\right|_{\Gamma_{1}}=0$. This allows to choose $v=-w^{+} \in V_{0}$ in problem (11) to obtain

$$
a\left(u_{\alpha}-u_{\infty},-w^{+}\right)+a\left(u_{\infty},-w^{+}\right)+\alpha \int_{\Gamma_{3}} j^{0}\left(u_{\alpha} ;-w^{+}\right) d \Gamma \geq L\left(-w^{+}\right) .
$$

Exploiting the relation $a\left(u_{\alpha}-u_{\infty},-w^{+}\right)=-a\left(w^{+}, w^{+}\right)$, we have

$$
-a\left(w^{+}, w^{+}\right)+a\left(u_{\infty},-w^{+}\right)+\alpha \int_{\Gamma_{3}} j^{0}\left(u_{\alpha} ;-w^{+}\right) d \Gamma \geq L\left(-w^{+}\right) .
$$

Next, part (a) of the proof shows that

$$
\left.w\right|_{\Gamma_{3}}=\left.\left(u_{\alpha}-b\right)\right|_{\Gamma_{3}} \leq 0
$$

and $\left.w^{+}\right|_{\Gamma_{3}}=0$, and consequently $w^{+} \in K_{0}$. Since $u_{\infty} \in K$ solves (12), taking $v=w^{+} \in K_{0}$ in equality (12), and using the result in (15), it follows that

$$
a\left(w^{+}, w^{+}\right) \leq \alpha \int_{\Gamma_{3}} j^{0}\left(u_{\alpha} ;-w^{+}\right) d \Gamma .
$$

Since $u_{\infty}=b$ on $\Gamma_{3}$, by (14), we get

$$
j^{0}\left(x, u_{\alpha} ;-\left(u_{\alpha}-u_{\infty}\right)^{+}\right)=j^{0}\left(x, u_{\alpha} ;-\left(u_{\alpha}-b\right)^{+}\right) \leq 0 \text { a.e. on } \Gamma_{3} \text {. }
$$

Again, by the coercivity of the form $a$, we have $m_{a}\left\|w^{+}\right\|_{V}^{2} \leq 0$. Therefore, $w^{+}=0$ in $\Omega$, and finally $u_{\alpha} \leq u_{\infty}$ in $\Omega$. This completes the proof. 
Note that properties (a) and (b) of Theorem 5 obtained for the hemivariational inequality (11) have been provided for linear elliptic problem (3) in properties (ii) and (iii) of Theorem 1 .

In what follows, we comment on the monotonicity property analogous to condition (iv) stated for problem (3) in Theorem 1.

Proposition 6 Assume that $H(j)$ and $\left(H_{0}\right)$ hold, and

$$
j^{0}\left(x, r ;-(r-s)^{+}\right)+c j^{0}\left(x, s ;(r-s)^{+}\right) \leq 0
$$

for all $c \geq 1$, all $r, s \in \mathbb{R}$, a.e. $x \in \Gamma_{3}$. Let $u_{\alpha_{i}} \in V_{0}$ denote the unique solution to the inequality (11) corresponding to $\alpha_{i}>0, i=1$, 2. Then the following monotonicity property holds:

$$
\alpha_{1} \leq \alpha_{2} \Longrightarrow u_{\alpha_{1}} \leq u_{\alpha_{2}} \text { in } \Omega
$$

Proof Let $0<\alpha_{1} \leq \alpha_{2}$ and $w=u_{\alpha_{1}}-u_{\alpha_{2}}$ in $\Omega$. It is sufficient to prove that $w^{+}=0$ in $\Omega$. Since $\left.w\right|_{\Gamma_{1}}=0$, we have $w^{+} \in V_{0}$. We choose $v=-w^{+} \in V_{0}$ in problem (11) for $\alpha_{1}$, and $v=w^{+} \in V_{0}$ in problem (11) for $\alpha_{2}$ to get

$$
\begin{gathered}
a\left(u_{\alpha_{1}},-w^{+}\right)+\alpha_{1} \int_{\Gamma_{3}} j^{0}\left(u_{\alpha_{1}} ;-w^{+}\right) d \Gamma \geq L\left(-w^{+}\right), \\
a\left(u_{\alpha_{2}}, w^{+}\right)+\alpha_{2} \int_{\Gamma_{3}} j^{0}\left(u_{\alpha_{2}} ; w^{+}\right) d \Gamma \geq L\left(w^{+}\right) .
\end{gathered}
$$

By adding the last two inequalities, we have

$$
-a\left(w, w^{+}\right)+\alpha_{1} \int_{\Gamma_{3}} j^{0}\left(u_{\alpha_{1}} ;-w^{+}\right) d \Gamma+\alpha_{2} \int_{\Gamma_{3}} j^{0}\left(u_{\alpha_{2}} ; w^{+}\right) d \Gamma \geq 0
$$

which implies

$$
\begin{aligned}
a\left(w^{+}, w^{+}\right) & \leq \int_{\Gamma_{3}}\left(\alpha_{1} j^{0}\left(u_{\alpha_{1}} ;-w^{+}\right)+\alpha_{2} j^{0}\left(u_{\alpha_{2}} ; w^{+}\right)\right) d \Gamma \\
& =\alpha_{1} \int_{\Gamma_{3}}\left(j^{0}\left(u_{\alpha_{1}} ;-w^{+}\right)+\frac{\alpha_{2}}{\alpha_{1}} j^{0}\left(u_{\alpha_{2}} ; w^{+}\right)\right) d \Gamma \leq 0 .
\end{aligned}
$$

Using the coercivity of the form $a$, we deduce that $w^{+}=0$, which completes the proof.

It is easy to check that the following two simple examples satisfy $H(j)$ and the condition (16):

$$
j(r)=\left\{\begin{array}{ll}
b-r & \text { for } r<b, \\
0 & \text { for } r \geq b,
\end{array} \text { and } \quad j(r)= \begin{cases}(b-r)^{2}+1 & \text { for } r<b, \\
1 & \text { for } r \geq b\end{cases}\right.
$$


Note also that hypothesis (16) implies that the function $j(x, \cdot)$ is convex for a.e. $x \in \Gamma_{3}$. In fact, if $r>s$, then $(r-s)^{+}=r-s$ and (16) gives

$$
j^{0}(x, r ; s-r)+c j^{0}(x, s ; r-s) \leq 0 \text { for all } c \geq 1 .
$$

In particular, taking $c=1$ we obtain the condition equivalent to the relaxed monotonicity condition with $m_{j}=0$, which means that $j(x, \cdot)$ is convex (see Remark 10).

We conclude that the monotonicity property of Proposition 6 holds for convex potentials, i.e., for variational inequalities. The proof of the monotonicity property for hemivariational inequalities remains an open problem.

\section{Asymptotic Behavior of Solutions}

In this section we investigate the asymptotic behavior of solutions to problem (11) when $\alpha \rightarrow \infty$. To this end, we need the following additional hypothesis on the superpotential $j$.

$\left(H_{1}\right): \quad$ if $j^{0}(x, r ; b-r)=0$ for all $r \in \mathbb{R}$, a.e. $x \in \Gamma_{3}$, then $r=b$.

Theorem 7 Assume $H(j),\left(H_{0}\right)$ and $\left(H_{1}\right)$. Let $\left\{u_{\alpha}\right\} \subset V_{0}$ be a sequence of solutions to problem (11) and $u_{\infty} \in K$ be the unique solution to problem (12). Then $u_{\alpha} \rightarrow u_{\infty}$ in $V$, as $\alpha \rightarrow \infty$.

Proof First, we prove the estimate on the sequence $\left\{u_{\alpha}\right\}$ in $V$. We choose $v=u_{\infty}-$ $u_{\alpha} \in V_{0}$ as a test function in problem (11) to obtain

$$
a\left(u_{\alpha}, u_{\infty}-u_{\alpha}\right)+\alpha \int_{\Gamma_{3}} j^{0}\left(u_{\alpha} ; u_{\infty}-u_{\alpha}\right) d \Gamma \geq L\left(u_{\infty}-u_{\alpha}\right) .
$$

From the equality $a\left(u_{\alpha}, u_{\infty}-u_{\alpha}\right)=-a\left(u_{\infty}-u_{\alpha}, u_{\infty}-u_{\alpha}\right)+a\left(u_{\infty}, u_{\infty}-u_{\alpha}\right)$, we get

$$
a(v, v)-\alpha \int_{\Gamma_{3}} j^{0}\left(u_{\alpha} ; v\right) d \Gamma \leq a\left(u_{\infty}, v\right)-L(v) .
$$

We observe that $j^{0}\left(x, u_{\alpha} ; v\right)=j^{0}\left(x, u_{\alpha} ; b-u_{\alpha}\right)$ on $\Gamma_{3}$, and by $H(j)(\mathrm{d})$, we have $j^{0}\left(x, u_{\alpha} ; v\right) \leq 0$ on $\Gamma_{3}$. Hence

$$
a(v, v) \leq a\left(u_{\infty}, v\right)-L(v)
$$

By the boundedness and coercivity of $a$, we infer

$$
m_{a}\|v\|_{V}^{2} \leq\left(M\left\|u_{\infty}\right\|_{V}+\|L\|_{V^{*}}\right)\|v\|_{V}
$$

with $M>0$, and subsequently

$$
\left\|u_{\alpha}\right\|_{V} \leq\|v\|_{V}+\left\|u_{\infty}\right\|_{V} \leq \frac{1}{m_{a}}\left(M\left\|u_{\infty}\right\|_{V}+\|L\|_{V^{*}}\right)+\left\|u_{\infty}\right\|_{V}=: C,
$$


where $C>0$ is independent of $\alpha$. Hence, since $a(v, v) \geq 0$, from (17), we have

$$
-\alpha \int_{\Gamma_{3}} j^{0}\left(u_{\alpha} ; v\right) d \Gamma \leq\left(M\left\|u_{\infty}\right\|_{V}+\|L\|_{V^{*}}\right)\|v\|_{V} \leq \frac{1}{m_{a}}\left(M\left\|u_{\infty}\right\|_{V}+\|L\|_{V^{*}}\right)^{2}=: C_{1},
$$

where $C_{1}>0$ is independent of $\alpha$. Thus

$$
-\int_{\Gamma_{3}} j^{0}\left(u_{\alpha} ; v\right) d \Gamma \leq \frac{C_{1}}{\alpha}
$$

It follows from (18) that $\left\{u_{\alpha}\right\}$ remains in a bounded subset of $V$. Thus, there exists $u^{*} \in V$ such that, by passing to a subsequence if necessary, we have

$$
u_{\alpha} \rightarrow u^{*} \text { weakly in } V \text {, as } \alpha \rightarrow \infty \text {. }
$$

Next, we show that $u^{*}=u_{\infty}$. We observe that $u^{*} \in V_{0}$ because $\left\{u_{\alpha}\right\} \subset V_{0}$ and $V_{0}$ is sequentially weakly closed in $V$. Let $w \in K$ and $v=w-u_{\alpha} \in V_{0}$. From (11), we have

$$
L\left(w-u_{\alpha}\right) \leq a\left(u_{\alpha}, w-u_{\alpha}\right)+\alpha \int_{\Gamma_{3}} j^{0}\left(u_{\alpha} ; w-u_{\alpha}\right) d \Gamma .
$$

Since $w=b$ on $\Gamma_{3}$, by $H(j)(\mathrm{d})$, we have

$$
\alpha \int_{\Gamma_{3}} j^{0}\left(u_{\alpha} ; w-u_{\alpha}\right) d \Gamma=\alpha \int_{\Gamma_{3}} j^{0}\left(u_{\alpha} ; b-u_{\alpha}\right) d \Gamma \leq 0
$$

which implies

$$
L\left(w-u_{\alpha}\right) \leq a\left(u_{\alpha}, w-u_{\alpha}\right)
$$

Next, we use the weak lower semicontinuity of the functional $V \ni v \mapsto a(v, v) \in \mathbb{R}$ and from (21), we deduce

$$
u^{*} \in V_{0} \text { satisfies } L\left(w-u^{*}\right) \leq a\left(u^{*}, w-u^{*}\right) \text { for all } w \in K
$$

Subsequently, we show that $u^{*} \in K$. In fact, from (20), by the compactness of the trace operator, we have $\left.\left.u_{\alpha}\right|_{\Gamma_{3}} \rightarrow u^{*}\right|_{\Gamma_{3}}$ in $L^{2}\left(\Gamma_{3}\right)$, as $\alpha \rightarrow \infty$. Passing to a subsequence if necessary, we may suppose that $u_{\alpha}(x) \rightarrow u^{*}(x)$ for a.e. $x \in \Gamma_{3}$ and there exists $h \in L^{2}\left(\Gamma_{3}\right)$ such that $\left|u_{\alpha}(x)\right| \leq h(x)$ a.e. $x \in \Gamma_{3}$. Using the upper semicontinuity of the function $\mathbb{R} \times \mathbb{R} \ni(r, s) \mapsto j^{0}(x, r ; s) \in \mathbb{R}$ for a.e. $x \in \Gamma_{3}$, see Proposition 3(iii), we get

$\lim \sup j^{0}\left(x, u_{\alpha}(x) ; u_{\infty}(x)-u_{\alpha}(x)\right) \leq j^{0}\left(x, u^{*}(x) ; u_{\infty}(x)-u^{*}(x)\right)$ a.e. $x \in \Gamma_{3}$. 
Next, taking into account the estimate

$\left|j^{0}\left(x, u_{\alpha}(x) ; u_{\infty}(x)-u_{\alpha}(x)\right)\right| \leq\left(c_{0}+c_{1}\left|u_{\alpha}(x)\right|\right)\left|b-u_{\alpha}(x)\right| \leq k(x)$ a.e. $x \in \Gamma_{3}$

with $k \in L^{1}\left(\Gamma_{3}\right)$ given by $k(x)=\left(c_{0}+c_{1} h(x)\right)(|b|+h(x))$, by the dominated convergence theorem, see [8, Theorem 2.2.33], we obtain

$$
\limsup \int_{\Gamma_{3}} j^{0}\left(u_{\alpha} ; u_{\infty}-u_{\alpha}\right) d \Gamma \leq \int_{\Gamma_{3}} j^{0}\left(u^{*} ; u_{\infty}-u^{*}\right) d \Gamma .
$$

Consequently, from $H(j)(\mathrm{d})$ and (19), we have

$$
0 \leq-\int_{\Gamma_{3}} j^{0}\left(u^{*} ; b-u^{*}\right) d \Gamma \leq \liminf \left(-\int_{\Gamma_{3}} j^{0}\left(u_{\alpha} ; u_{\infty}-u_{\alpha}\right) d \Gamma\right) \leq 0
$$

which gives $\int_{\Gamma_{3}} j^{0}\left(u^{*} ; b-u^{*}\right) d \Gamma=0$. Again by $H(j)(\mathrm{d})$, we get $j^{0}\left(x, u^{*} ; b-u^{*}\right)=$ 0 a.e. $x \in \Gamma_{3}$. Using $\left(H_{1}\right)$, we have $u^{*}(x)=b$ for a.e. $x \in \Gamma_{3}$, which together with (22) implies

$$
u^{*} \in K \text { satisfies } L\left(w-u^{*}\right) \leq a\left(u^{*}, w-u^{*}\right) \text { for all } w \in K .
$$

Next, we prove that $u^{*}=u_{\infty}$. To this end, let $v:=w-u^{*} \in K_{0}$ with arbitrary $w \in K$. Hence, $L(v) \leq a\left(u^{*}, v\right)$ for all $v \in K_{0}$. Recalling that $v \in K_{0}$ implies $-v \in K_{0}$, we obtain $a\left(u^{*}, v\right) \leq L(v)$ for all $v \in K_{0}$. Hence, we conclude that

$$
u^{*} \in K \text { satisfies } a\left(u^{*}, v\right)=L(v) \text { for all } v \in K_{0} \text {, }
$$

i.e., $u^{*} \in K$ is a solution to problem (12). By the uniqueness of solution to problem (12), we have $u^{*}=u_{\infty}$ and hence $u_{\alpha} \rightarrow u_{\infty}$ weakly in $V$, as $\alpha \rightarrow \infty$. From the uniqueness of solution to (12), we also infer that the whole sequence $\left\{u_{\alpha}\right\}$ converges weakly in $V$ to $u_{\infty}$.

Finally, we prove the strong convergence $u_{\alpha} \rightarrow u_{\infty}$ in $V$, as $\alpha \rightarrow \infty$. Choosing $v=u_{\infty}-u_{\alpha} \in V_{0}$ in problem (11), we obtain

$$
a\left(u_{\alpha}, u_{\infty}-u_{\alpha}\right)+\alpha \int_{\Gamma_{3}} j^{0}\left(u_{\alpha} ; u_{\infty}-u_{\alpha}\right) d \Gamma \geq L\left(u_{\infty}-u_{\alpha}\right) .
$$

Hence

$$
\begin{aligned}
& a\left(u_{\infty}-u_{\alpha}, u_{\infty}-u_{\alpha}\right) \leq a\left(u_{\infty}, u_{\infty}-u_{\alpha}\right)+L\left(u_{\alpha}-u_{\infty}\right) \\
& \quad+\alpha \int_{\Gamma_{3}} j^{0}\left(u_{\alpha} ; u_{\infty}-u_{\alpha}\right) d \Gamma .
\end{aligned}
$$

Since $u_{\infty}=b$ on $\Gamma_{3}$, by $H(j)(\mathrm{d})$ and the coercivity of the form $a$, we have

$$
m_{a}\left\|u_{\infty}-u_{\alpha}\right\|_{V}^{2} \leq a\left(u_{\infty}, u_{\infty}-u_{\alpha}\right)+L\left(u_{\alpha}-u_{\infty}\right) .
$$


Employing the weak continuity of both $a\left(u_{\infty}, \cdot\right)$ and $L$, we conclude that $u_{\alpha} \rightarrow u_{\infty}$ in $V$, as $\alpha \rightarrow \infty$. This completes the proof.

\section{Continuous Dependence Result}

In this section we provide the result on continuous dependence of solution to problem (11) on the internal energy $g$ and the heat flux $q$ for fixed $\alpha>0$.

First, from the compactness of the embedding $V$ into $L^{2}(\Omega)$ and of the trace operator from $V$ into $L^{2}(\Gamma)$, we obtain the following convergence result.

Lemma 8 Let $g_{n} \in L^{2}(\Omega), q_{n} \in L^{2}\left(\Gamma_{2}\right)$ for $n \in \mathbb{N}$. Define $L_{n} \in V^{*}, n \in \mathbb{N}$, by

$$
L_{n}(v)=\int_{\Omega} g_{n} v d x-\int_{\Gamma_{2}} q_{n} v d \Gamma \text { for } v \in V
$$

If $g_{n} \rightarrow g$ weakly in $L^{2}(\Omega), q_{n} \rightarrow q$ weakly in $L^{2}\left(\Gamma_{2}\right)$, and $v_{n} \in V, v_{n} \rightarrow v$ weakly in $V$, then

$$
L_{n}\left(v_{n}\right) \rightarrow L(v), \text { as } n \rightarrow \infty
$$

and there exists a constant $C>0$ independent of $n$ such that $\left\|L_{n}\right\|_{V^{*}} \leq C$ for all $n \in \mathbb{N}$.

The continuous dependence result reads as follows.

Theorem 9 Assume that $\alpha>0$ is fixed, $L, L_{n} \in V^{*}, n \in \mathbb{N}$ and $H(j)$ holds. Let $u_{n} \in V_{0}, n \in \mathbb{N}$, be a solution to problem (11) corresponding to $L_{n}$, and

$$
\lim L_{n}\left(z_{n}\right)=L(z) \text { for any } z_{n} \rightarrow z \text { weakly in } V \text {, as } n \rightarrow \infty \text {. }
$$

Then, there exists a subsequence of $\left\{u_{n}\right\}$ which converges weakly in $V$ to a solution of problem (11) corresponding to L. If, in addition, the following hypotheses hold

$$
\begin{aligned}
& j^{0}(x, r ; s-r)+j^{0}(x, s ; r-s) \leq m_{j}|r-s|^{2} \text { for all } r, s \in \mathbb{R} \text {, a.e. } x \in \Gamma_{3}, \\
& \quad m_{a}>\alpha m_{j}\|\gamma\|^{2},
\end{aligned}
$$

where $m_{j} \geq 0$, then problem (11) has the unique solution $u$ and $u_{n} \in V_{0}$ corresponding to $L$ and $L_{n}$, respectively, and the whole sequence $\left\{u_{n}\right\}$ converges to $u$ in $V$, as $n \rightarrow \infty$.

Proof Let $u_{n} \in V_{0}$ be a solution to problem (11) corresponding to $L_{n}$, and $u_{\infty} \in K$ be the solution to problem (12). We have

$$
a\left(u_{n}, u_{\infty}-u_{n}\right)+\alpha \int_{\Gamma_{3}} j^{0}\left(u_{n} ; u_{\infty}-u_{n}\right) d \Gamma \geq L_{n}\left(u_{\infty}-u_{n}\right) .
$$


Hence

$$
\begin{aligned}
& a\left(u_{\infty}-u_{n}, u_{\infty}-u_{n}\right) \leq a\left(u_{\infty}, u_{\infty}-u_{n}\right)+L_{n}\left(u_{n}-u_{\infty}\right) \\
& \quad+\alpha \int_{\Gamma_{3}} j^{0}\left(u_{n} ; b-u_{n}\right) d \Gamma .
\end{aligned}
$$

From hypothesis $H(j)(\mathrm{d})$, since the form $a$ is bounded and coercive, we get

$$
\begin{aligned}
m_{a}\left\|u_{\infty}-u_{n}\right\|_{V}^{2} & \leq a\left(u_{\infty}, u_{\infty}-u_{n}\right)+L_{n}\left(u_{n}-u_{\infty}\right) \\
& \leq M\left\|u_{\infty}\right\|_{V}\left\|u_{\infty}-u_{n}\right\|_{V}+\left\|L_{n}\right\|_{V^{*}}\left\|u_{\infty}-u_{n}\right\|_{V},
\end{aligned}
$$

and subsequently

$$
\left\|u_{n}\right\|_{V} \leq\left\|u_{\infty}-u_{n}\right\|_{V}+\left\|u_{\infty}\right\|_{V} \leq \frac{1}{m_{a}}\left(M\left\|u_{\infty}\right\|_{V}+k_{1}\right)+\left\|u_{\infty}\right\|_{V} \leq k_{2}
$$

for all $n \in \mathbb{N}$ with $k_{1}, k_{2}>0$ independent of $n$. Hence, $\left\{u_{n}\right\}$ is uniformly bounded in $V$ and also in $V_{0}$. From the reflexivity of $V_{0}$, there exist $\xi \in V_{0}$ and a subsequence of $\left\{u_{n}\right\}$, denoted in the same way, such that

$$
u_{n} \rightarrow \xi \text { weakly in } V_{0} \text {, as } n \rightarrow \infty \text {. }
$$

We show that $\xi \in V_{0}$ satisfies (11). We know that $u_{n} \in V_{0}$ and

$$
a\left(u_{n}, v\right)+\alpha \int_{\Gamma_{3}} j^{0}\left(u_{n} ; v\right) d \Gamma \geq L_{n}(v) \text { for all } v \in V_{0} .
$$

Taking the upper limit, we use the weak continuity of $a(\cdot, v)$ and $(23)$ to get

$$
a(\xi, v)+\alpha \limsup \int_{\Gamma_{3}} j^{0}\left(u_{n} ; v\right) d \Gamma \geq \lim L_{n}(v)=L(v) \text { for all } v \in V_{0}
$$

By the compactness of the trace operator from $V$ into $L^{2}\left(\Gamma_{3}\right)$, we have $\left.\left.u_{n}\right|_{\Gamma_{3}} \rightarrow \xi\right|_{\Gamma_{3}}$ in $L^{2}\left(\Gamma_{3}\right)$, as $n \rightarrow \infty$, and at least for a subsequence, $u_{n}(x) \rightarrow \xi(x)$ for a.e. $x \in \Gamma_{3}$ and $\left|u_{n}(x)\right| \leq \eta(x)$ a.e. $x \in \Gamma_{3}$, where $\eta \in L^{2}\left(\Gamma_{3}\right)$. Since the function $\mathbb{R} \times \mathbb{R} \ni$ $(r, s) \mapsto j^{0}(x, r ; s) \in \mathbb{R}$ a.e. on $\Gamma_{3}$ is upper semicontinuous, see Proposition 3(iii), we obtain

$$
\limsup j^{0}\left(x, u_{n}(x) ; v(x)\right) \leq j^{0}(x, \xi(x) ; v(x)) \text { a.e. } x \in \Gamma_{3} \text {. }
$$

Recalling the estimate

$$
\left|j^{0}\left(x, u_{n}(x) ; v(x)\right)\right| \leq\left(c_{0}+c_{1}\left|u_{n}(x)\right|\right)|v(x)| \leq k(x) \text { a.e. } x \in \Gamma_{3}
$$


where $k \in L^{1}\left(\Gamma_{3}\right), k(x)=\left(c_{0}+c_{1} \eta(x)\right)|v(x)|$, we apply the dominated convergence theorem, see [8, Theorem 2.2.33] to get

$$
\limsup \int_{\Gamma_{3}} j^{0}\left(u_{n} ; v\right) d \Gamma \leq \int_{\Gamma_{3}} \lim \sup j^{0}\left(u_{n} ; v\right) d \Gamma \leq \int_{\Gamma_{3}} j^{0}(\xi ; v) d \Gamma .
$$

Using the latter in (26) entails

$$
a(\xi, v)+\alpha \int_{\Gamma_{3}} j^{0}(\xi ; v) d \Gamma \geq L(v) \text { for all } v \in V_{0}
$$

which means that $\xi \in V_{0}$ is a solution to problem (11), and completes the first part of the proof.

Next, in addition, we assume (24) and (25). The existence of solution to (11) follows from the first part of the theorem. To prove uniqueness, let $u_{1}, u_{2} \in V_{0}$ solve (11). Then taking as test functions $u_{2}-u_{1} \in V_{0}$ for $u_{1}$ and $u_{1}-u_{2} \in V_{0}$ for $u_{2}$, and adding corresponding inequalities, we obtain

$$
a\left(u_{1}-u_{2}, u_{2}-u_{1}\right)+\alpha \int_{\Gamma_{3}}\left(j^{0}\left(u_{1} ; u_{2}-u_{1}\right)+j^{0}\left(u_{2} ; u_{1}-u_{2}\right)\right) d \Gamma \geq 0 .
$$

From the coercivity of the form $a$ and (24), we have

$$
m_{a}\left\|u_{1}-u_{2}\right\|_{V}^{2} \leq \alpha m_{j} \int_{\Gamma_{3}}\left|u_{1}(x)-u_{2}(x)\right|^{2} d \Gamma \leq \alpha m_{j}\|\gamma\|^{2}\left\|u_{1}-u_{2}\right\|_{V}^{2} .
$$

Since $\left(m_{a}-\alpha m_{j}\|\gamma\|^{2}\right)\left\|u_{1}-u_{2}\right\|_{V}^{2} \leq 0$, and by the smallness condition (25), we get $u_{1}=u_{2}$. Hence, we deduce that solutions $u, u_{n} \in V_{0}$ to (11) are unique, and by (27), we immediately have $\xi=u$.

Finally, we show the strong convergence of $\left\{u_{n}\right\}$ to $u$ in $V$. We choose suitable test functions from $V_{0}$ in (11) and (27) to obtain

$$
\begin{gathered}
a\left(u_{n}, u-u_{n}\right)+\alpha \int_{\Gamma_{3}} j^{0}\left(u_{n} ; u-u_{n}\right) d \Gamma \geq L_{n}\left(u-u_{n}\right) \\
a\left(u, u_{n}-u\right)+\alpha \int_{\Gamma_{3}} j^{0}\left(u ; u_{n}-u\right) d \Gamma \geq L\left(u_{n}-u\right) .
\end{gathered}
$$

Adding the two inequalities, we have

$$
\begin{aligned}
& a\left(u_{n}-u, u-u_{n}\right)+\alpha \int_{\Gamma_{3}}\left(j^{0}\left(u_{n} ; u-u_{n}\right)+j^{0}\left(u ; u_{n}-u\right)\right) d \Gamma \\
& \quad \geq L_{n}\left(u-u_{n}\right)+L\left(u_{n}-u\right) .
\end{aligned}
$$

Using the coercivity of the form $a$ and (24), we get

$$
m_{a}\left\|u_{n}-u\right\|_{V}^{2} \leq \alpha m_{j}\|\gamma\|^{2}\left\|u_{n}-u\right\|_{V}^{2}+L_{n}\left(u_{n}-u\right)+L\left(u-u_{n}\right)
$$


which entails

$$
\left(m_{a}-\alpha m_{j}\|\gamma\|^{2}\right)\left\|u_{n}-u\right\|_{V}^{2} \leq L_{n}\left(u_{n}-u\right)+L\left(u-u_{n}\right) .
$$

From hypotheses (23) and (25), we deduce that $\left\|u_{n}-u\right\|_{V} \rightarrow 0$, as $n \rightarrow \infty$. Since $u \in V_{0}$ is unique, we infer that the whole sequence $\left\{u_{n}\right\}$ converges in $V$ to $u$. This proof is complete.

Remark 10 It is known that for a locally Lipschitz function $j: \mathbb{R} \rightarrow \mathbb{R}$, the condition (24) is equivalent to the so-called relaxed monotonicity condition of the subdifferential

$$
\left(\eta_{1}-\eta_{2}\right)\left(r_{1}-r_{2}\right) \geq-m_{j}\left|r_{1}-r_{2}\right|^{2}
$$

for all $r_{i} \in \mathbb{R}, \eta_{i} \in \partial j\left(r_{i}\right), i=1,2$. The latter was extensively used in the literature, see [17] and the references therein. Condition (24) can be verified by proving that the function

$$
\mathbb{R} \ni r \mapsto j(r)+\frac{m_{j}}{2}|r|^{2} \in \mathbb{R}
$$

is convex. An example of a nonconvex function which satisfies the condition (24) is given in Example 11. Note that if $j: \mathbb{R} \rightarrow \mathbb{R}$ is convex, then (24) and (28) hold with $m_{j}=0$. In fact, by convexity,

$$
j^{0}(r ; s-r) \leq j(s)-j(r) \text { and } j^{0}(s ; r-s) \leq j(r)-j(s)
$$

for all $r, s \in \mathbb{R}$ which imply $j^{0}(r ; s-r)+j^{0}(s ; r-s) \leq 0$. Therefore, for a convex function $j: \mathbb{R} \rightarrow \mathbb{R}$, condition (24) or, equivalently, (28) reduces to monotonicity of the (convex) subdifferential, i.e., $m_{j}=0$.

\section{Examples}

The following examples provide nonconvex and convex functions which satisfies the hypotheses $H(j),\left(H_{1}\right)$ and (24).

Example 11 Let $j: \mathbb{R} \rightarrow \mathbb{R}$ be the function defined by

$$
j(r)=\left\{\begin{array}{cc}
(r-b)^{2} & \text { if } r<b, \\
1-e^{-(r-b)} & \text { if } r \geq b
\end{array}\right.
$$

for $r \in \mathbb{R}$ with a constant $b \in \mathbb{R}$. This function is nonconvex, locally Lipschitz and its subdifferential is given by

$$
\partial j(r)=\left\{\begin{array}{cc}
2(r-b) & \text { if } r<b \\
{[0,1]} & \text { if } r=b, \\
e^{-(r-b)} & \text { if } r>b
\end{array}\right.
$$


for all $r \in \mathbb{R}$. Hence, we have $|\partial j(r)| \leq 1+2|b|+2|r|$ for all $r \in \mathbb{R}$. Moreover, using Proposition 3(ii), one has

$$
j^{0}(r ; b-r)=\max \{\zeta(b-r) \mid \zeta \in \partial j(r)\}=\left\{\begin{array}{cl}
-2(b-r)^{2} & \text { if } r<b \\
0 & \text { if } r=b \\
e^{-(r-b)}(b-r) & \text { if } r>b
\end{array}\right.
$$

for all $r \in \mathbb{R}$. Thus $H(j)$ is satisfied. By the above formula, we also infer that $\left(H_{1}\right)$ is satisfied. Further, we show that condition (24) holds with $m_{j}=1$. The condition (24) is equivalent to the relaxed monotonicity of the subdifferential

$$
(\partial j(r)-\partial j(s))(r-s) \geq-|r-s|^{2} \text { for all } r, s \in \mathbb{R} \text {. }
$$

The latter means that

$$
\left(\partial\left(j(r)+\frac{1}{2} r^{2}\right)-\partial\left(\left(j(s)+\frac{1}{2} s^{2}\right)\right)(r-s) \geq 0 \text { for all } r, s \in \mathbb{R},\right.
$$

i.e., the subdifferential $\partial \psi$ of the function $\psi: \mathbb{R} \rightarrow \mathbb{R}$ defined by $\psi(r)=j(r)+\frac{1}{2} r^{2}$ is monotone. Now, the monotonicity of $\partial \psi$ can be verified using the formula

$$
\partial \psi(r)=\left\{\begin{array}{cc}
3 r-2 b & \text { if } r<b \\
{[b, b+1]} & \text { if } r=b \\
e^{-(r-b)}+r & \text { if } r>b
\end{array}\right.
$$

for all $r \in \mathbb{R}$. We conclude that $H(j),\left(H_{1}\right)$ and (24) are satisfied.

Example 12 (see [15, Example 3]) Let the function $j: \mathbb{R} \rightarrow \mathbb{R}$ be given by

$$
j(r)=\min \left\{j_{1}(r), j_{2}(r)\right\}
$$

for $r \in \mathbb{R}$, where $j_{i}: \mathbb{R} \rightarrow \mathbb{R}$ are convex, quadratic and such that $j_{i}^{\prime}(b)=0, i=1,2$. It is known, see [6, Theorem 2.5.1], that

$$
\partial j(r) \subset \operatorname{conv}\left\{j_{1}^{\prime}(r), j_{2}^{\prime}(r)\right\} \text { for all } r \in \mathbb{R},
$$

so, the subgradient of $j$ has at most a linear growth. Using the monotonicity of the subgradient of convex function, we get

$$
0 \leq\left(j_{i}^{\prime}(b)-j_{i}^{\prime}(r)\right)(b-r)=-j_{i}^{\prime}(r)(b-r) \text { for all } r \in \mathbb{R}, i=1,2,
$$

and, by Proposition 3(iii), we have

$$
\begin{aligned}
j^{0}(r ; b-r) & =\max \{\zeta(b-r) \mid \zeta \in \partial j(r)\} \\
& =\max \left\{\left(\lambda j_{1}^{\prime}(r)+(1-\lambda) j_{2}^{\prime}(r)\right)(b-r) \mid \lambda \in[0,1]\right\} \leq 0 .
\end{aligned}
$$


Hence, we deduce that condition $H(j)$ is satisfied. Similarly, if $j^{0}(r ; b-r)=0$ for all $r \in \mathbb{R}$, then $\lambda j_{1}^{\prime}(r)(b-r)=0$ and $(1-\lambda) j_{2}^{\prime}(r)(b-r)=0$ for all $r \in \mathbb{R}$ with $\lambda \in[0,1]$, which is possible when $r=b$. So, $j$ satisfies also $\left(H_{1}\right)$. Further, it is easy to observe that in the case when the graphs of functions $j_{1}$ and $j_{2}$ have two common points, then the function $j$ is nonconvex.

Example 13 Let $j: \mathbb{R} \rightarrow \mathbb{R}$ be the function defined by

$$
j(r)=\frac{1}{2}(r-b)^{2}
$$

for $r \in \mathbb{R}$ with $b \in \mathbb{R}$. Then

$$
j^{0}(r ; s)=(r-b) s \text { and } \partial j(r)=r-b
$$

for $r, s \in \mathbb{R}$. Moreover, we have $j^{0}(r ; b-r)=(r-b)(b-r)=-(b-r)^{2} \leq 0$ for all $r \in \mathbb{R}$. Also, for all $r \in \mathbb{R}$, if $j^{0}(r ; b-r)=0$, then $(r-b)(b-r)=-(b-r)^{2}=0$, which implies $r=b$. Hence we deduce that $j$ satisfies properties $H(j)$ and $\left(H_{1}\right)$. By Remark 10, it is clear that $j$ satisfies (24) with $m_{j}=0$.

Example 14 Let $m_{1}, m_{2}, r_{0} \in \mathbb{R}$ be constants such that $m_{1} \leq-r_{0}<0$ and $m_{2} \geq r_{0}>$ 0 . Consider the function $j: \mathbb{R} \rightarrow \mathbb{R}$ defined by

$$
j(r)= \begin{cases}\frac{r_{0}^{2}}{2}+m_{1}\left[r-\left(b-r_{0}\right)\right] & \text { if } r<b-r_{0}, \\ \frac{1}{2}(r-b)^{2} & \text { if } b-r_{0} \leq r \leq b+r_{0}, \\ \frac{r_{0}^{2}}{2}+m_{2}\left[r-\left(b+r_{0}\right)\right] & \text { if } r>b+r_{0}\end{cases}
$$

for $r \in \mathbb{R}, b \in \mathbb{R}$. The function $j$ is convex, its subdifferential is given by

$$
\partial j(r)= \begin{cases}m_{1} & \text { if } r<b-r_{0}, \\ {\left[m_{1},-r_{0}\right]} & \text { if } r=b-r_{0}, \\ r-b & \text { if } b-r_{0}<r<b+r_{0}, \\ {\left[r_{0}, m_{2}\right]} & \text { if } r=b+r_{0}, \\ m_{2} & \text { if } r>b+r_{0}\end{cases}
$$

for all $r \in \mathbb{R}$, and its generalized directional derivative has the form

$$
j^{0}(r ; b-r)= \begin{cases}m_{1}(b-r)<0 & \text { if } r<b-r_{0}, \\ -r_{0}^{2}<0 & \text { if } r=b-r_{0}, \\ -(b-r)^{2} \leq 0 & \text { if } b-r_{0}<r<b+r_{0}, \\ -r_{0}^{2}<0 & \text { if } r=b+r_{0}, \\ m_{2}(b-r)<0 & \text { if } r>b+r_{0}\end{cases}
$$


for all $r, s \in \mathbb{R}$. Hence, we obtain that $j^{0}(r ; b-r) \leq 0$ for all $r \in \mathbb{R}$. Similarly, if $j^{0}(r ; b-r)=0$ for all $r \in \mathbb{R}$, then $r=b$. We conclude that $j$ satisfies $H(j)$ and $\left(H_{1}\right)$. Moreover, the function $j$, being convex, satisfies (24) with $m_{j}=0$, see Remark 10.

Example 15 We define $j: \mathbb{R} \rightarrow \mathbb{R}$ by

$$
j(r)=|r-b|= \begin{cases}-r+b & \text { if } r \leq b \\ r-b & \text { if } r>b\end{cases}
$$

for $r \in \mathbb{R}$ with a constant $b \in \mathbb{R}$. Then, we have

$$
\partial j(r)= \begin{cases}-1 & \text { if } r<b, \\ {[-1,1]} & \text { if } r=b, \\ 1 & \text { if } r>b\end{cases}
$$

for all $r \in \mathbb{R}$, and

$$
j^{0}(r ; b-r)= \begin{cases}b-r & \text { if } r>b, \\ 0 & \text { if } r=b, \\ r-b & \text { if } r<b\end{cases}
$$

for all $r \in \mathbb{R}$. Thus, $j^{0}(r ; b-r) \leq 0$ for all $r \in \mathbb{R}$. Also, we observe that if $j^{0}(r ; b-r)=0$ for all $r \in \mathbb{R}$, then $r=b$. In consequence, the properties $H(j)$ and $\left(H_{1}\right)$ are verified. Further, since $j$ is convex, it satisfies (24) with $m_{j}=0$, see Remark 10.

\section{Conclusions}

We have studied the nonlinear elliptic problem with mixed boundary conditions involving a nonmonotone multivalued subdifferential boundary condition on a part of the boundary. Based on the notion of the Clarke generalized gradient, the variational form of the problem leads to an elliptic boundary hemivariational inequality. We have provided results on existence, comparison of solutions and continuous dependence on the data. Sufficient conditions have been found which guarantee the asymptotic behavior of solution, when the heat transfer coefficient tends to infinity, to a problem with the Dirichlet boundary condition. Under our hypotheses, the proof of the monotonicity property of Theorem 1(iv) for the elliptic hemivariational inequality (7) remains an interesting open problem. We have also given some examples of locally Lipschitz (nondifferentiable and nonconvex) functions to which our results can be applied.

Open Access This article is licensed under a Creative Commons Attribution 4.0 International License, which permits use, sharing, adaptation, distribution and reproduction in any medium or format, as long as you give appropriate credit to the original author(s) and the source, provide a link to the Creative Commons licence, and indicate if changes were made. The images or other third party material in this article are included 
in the article's Creative Commons licence, unless indicated otherwise in a credit line to the material. If material is not included in the article's Creative Commons licence and your intended use is not permitted by statutory regulation or exceeds the permitted use, you will need to obtain permission directly from the copyright holder. To view a copy of this licence, visit http://creativecommons.org/licenses/by/4.0/.

\section{References}

1. Azzam, A., Kreyszig, E.: On solutions of elliptic equations satisfying mixed boundary conditions. SIAM J. Math. Anal. 13, 254-262 (1982)

2. Bacuta, C., Bramble, J.H., Pasciak, J.E.: Using finite element tools in proving shift theorems for elliptic boundary value problems. Numer. Linear Algebra Appl. 10, 33-64 (2003)

3. Barbu, V.: Boundary control problems with non linear state equation. SIAM J. Control Optim. 20, 125-143 (1982)

4. Boukrouche, M., Tarzia, D.A.: On existence, uniqueness, and convergence of optimal control problems governed by parabolic variational inequalities. In: Hömberg, D., Tröltzsch, F. (eds.) IFIP Advances in Information and Communication Technology 391, pp. 76-84. Springer, Berlin (2013)

5. Carl, S., Le, V.K., Motreanu, D.: Nonsmooth Variational Problems and Their Inequalities. Springer, New York (2007)

6. Clarke, F.H.: Optimization and Nonsmooth Analysis. Wiley Interscience, New York (1983)

7. Denkowski, Z., Migorski, S., Papageorgiou, N.S.: An Introduction to Nonlinear Analysis: Theory. Kluwer Academic, Boston (2003)

8. Denkowski, Z., Migorski, S., Papageorgiou, N.S.: An Introduction to Nonlinear Analysis: Applications. Kluwer Academic, Boston (2003)

9. Duvaut, G., Lions, J.L.: Les Inéquations en Mécanique et en Physique. Dunod, Paris (1972)

10. Garguichevich, G.G., Tarzia, D.A.: The steady-state two-fase Stefan problem with an internal energy and some related problems. Atti Sem. Mat. Fis. Univ. Modena 39, 615-634 (1991)

11. Gasinski, L., Liu, Z., Migorski, S., Ochal, A., Peng, Z.: Hemivariational inequality approach to evolutionary constrained problems on star-shaped sets. J. Optim. Theory Appl. 164, 514-533 (2015)

12. Grisvard, P.: Elliptic Problems in Nonsmooth Domains. Pitman, London (1985)

13. Kawohl, B.: On nonlinear parabolic equations with abruptly changing nonlinear boundary conditions. Nonlinear Anal. 5, 1141-1153 (1981)

14. Lanzani, L., Capagna, L., Brown, R.M.: The mixed problem in $L^{p}$ for some two-dimensional Lipschitz domain. Math. Ann. 342, 91-124 (2008)

15. Migorski, S., Ochal, A.: Boundary hemivariational inequality of parabolic type. Nonlinear Anal. 57, 579-596 (2004)

16. Migorski, S., Ochal, A.: A unified approach to dynamic contact problems in viscoelasticity. J. Elasticity 83, 247-275 (2006)

17. Migorski, S., Ochal, A., Sofonea, M.: Nonlinear Inclusions and Hemivariational Inequalities, Models and Analysis of Contact Problems. Springer, New York (2013)

18. Migorski, S., Ochal, A., Sofonea, M.: A class of variational-hemivariational inequalities in reflexive Banach spaces. J. Elasticity 127, 151-178 (2017)

19. Naniewicz, Z., Panagiotopoulos, P.D.: Mathematical Theory of Hemivariational Inequalities and Applications. Marcel Dekker Inc., New York (1995)

20. Panagiotopoulos, P.D.: Nonconvex problems of semipermeable media and related topics. Z. Angew. Math. Mech. 65, 29-36 (1985)

21. Panagiotopoulos, P.D.: Inequality Problems in Mechanics and Applications. Birkhäuser, Boston (1985)

22. Panagiotopoulos, P.D.: Hemivariational Inequalities. Applications in Mechanics and Engineering. Springer, Berlin (1993)

23. Rodrigues, J.F.: Obstacle Problems in Mathematical Physics. North-Holland, Amsterdam (1987)

24. Shillor, M., Sofonea, M., Telega, J.J.: Models and Analysis of Quasistatic Contact. Springer, Berlin (2004)

25. Sofonea, M., Migorski, S.: Variational-Hemivariational Inequalities with Applications. CRC Press, Boca Raton (2018) 
26. Tabacman, E.D., Tarzia, D.A.: Sufficient and/or necessary condition for the heat transfer coefficient on $\Gamma_{1}$ and the heat flux on $\Gamma_{2}$ to obtain a steady-state two-phase Stefan problem. J. Differ. Equ. 77, 16-37 (1989)

27. Tarzia, D.A.: Sur le problème de Stefan à deux phases. C. R. Acad. Sci. Paris Ser. A 288, 941-944 (1979)

28. Tarzia, D.A.: Aplicación de métodos variacionales en el caso estacionario del problema de Stefan a dos fases. Math. Notae 27, 145-156 (1979-1980)

29. Tarzia, D.A.: Una familia de problemas que converge hacia el caso estacionario del problema de Stefan a dos fases. Math. Notae 27, 157-165 (1979-1980)

30. Tarzia, D.A.: An inequality for the constant heat flux to obtain a steady-state two-phase Stefan problem. Eng. Anal. 5(4), 177-181 (1988)

31. Zeidler, E.: Nonlinear Functional Analysis and Applications. II A/B. Springer, New York (1990)

32. Zeng, B., Liu, Z., Migorski, S.: On convergence of solutions to variational-hemivariational inequalities. Z. Angew. Math. Phys. 69(87), 1-20 (2018)

Publisher's Note Springer Nature remains neutral with regard to jurisdictional claims in published maps and institutional affiliations.

\section{Authors and Affiliations}

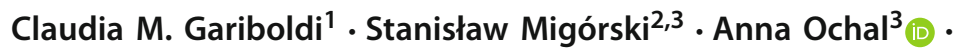 Domingo A. Tarzia ${ }^{4}$}

$凶 \quad$ Anna Ochal

anna.ochal@uj.edu.pl

Claudia M. Gariboldi

cgariboldi@exa.unrc.edu.ar

Stanisław Migórski

stanislaw.migorski@uj.edu.pl

Domingo A. Tarzia

DTarzia@austral.edu.ar

1 Depto. Matemática, FCEFQyN, Univ. Nac. de Río Cuarto, Ruta 36 Km 601, 5800 Río Cuarto, Argentina

2 College of Applied Mathematics, Chengdu University of Information Technology, Chengdu 610225, Sichuan, People's Republic of China

3 Chair of Optimization and Control, Jagiellonian University in Krakow, ul. Lojasiewicza 6, 30348 Krakow, Poland

4 Depto. Matemática-CONICET, FCE, Universidad Austral, Paraguay 1950, S2000FZF Rosario, Argentina 